\title{
Robot Manipulation of Deformable Objects
}

Edited by

Dominik Henrich and Heinz Wörn

Springer-Verlag London, Berlin, Heidelberg 


\section{Table of Contents}

Contributors ............................................................................................................................................. vi

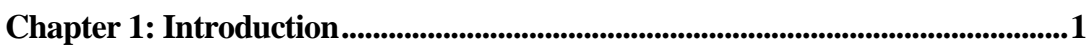

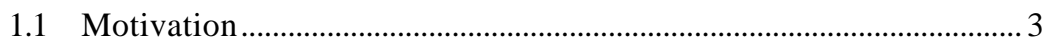

1.2 About this Book ............................................................................... 7

Chapter 2: Material Modeling and Simulation ...........................................................9

2.1 Energy-Based Modeling of Deformable Linear Objects ...................... 11

2.2 Discrete Element Approach for Non-Rigid Material

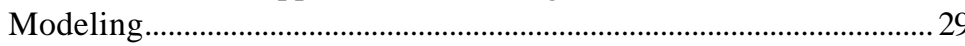

2.3 Direct and Inverse Simulation of Deformable Linear

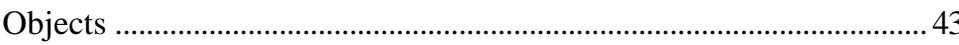

Chapter 3: Planning and Control Strategies ................................................................71

3.1 Indirect Simultaneous Positioning of Extensible

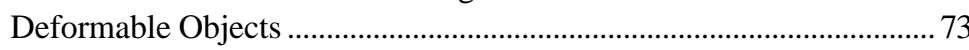

3.2 A Hybrid Position / Force Approach to the Exploitation of

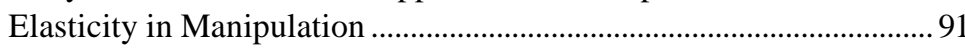

3.3 Force- and Vision-Based Detection of Contact State

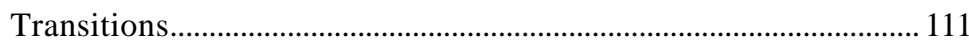

3.4 Automated Sewing System and Unfolding Fabric ........................... 135

Chapter 4: Collaborative Systems ............................................................................. 159

4.1 Manipulation of Sheet Metal by Dual Manipulators .......................... 161

4.2 A Manipulated Deformable Object as an Underactuated Mechanical System 175

Chapter 5: Applications and Industrial Experiences ........................................ 197

5.1 Simulation of Non-Rigid Materials Handling ....................................... 199

5.2 Robotics for Deheading White Fish ..................................................... 211

5.3 Application of LLW Robots to Distribution Lines............................. 237

5.4 Flexible Automatic Wiring of Long-Tube Lighting and Service Cabinet Modules 


\section{Contributors}

\section{Frank Abegg}

Institute for Process Control and Robotics (IPR)

Computer Science Department

Universität Karlsruhe (TH)

Kaiserstrasse 12

D-76128 Karlsruhe, Germany

Fax: +49-721-608-7141

E-mail: Abegg@ira.uka.de

Http://wwwipr.ira.uka.de/ paro/

\section{Monica Bordegoni}

Dipartimento di Ingegneria Industriale Universita' degli Studi di Parma

Parco Area delle Scienze 181/A

I-43100 Parma, Italia

Fax: +39-0521-905705

E-mail: mb@ied.unipr.it

Http://www.unipr.it/

\section{Rob Buckingham}

Oliver Crispin Consulting Ltd

5 Fallodon Way

Henleaze

Bristol BS9 4HR, United Kingdom

Fax: +44-117-983-4397

E-mail: rob@ocrobotics.co.uk

Http://www.ocrobotics.co.uk

\section{Giancarlo Frugoli}

Dipartimento di Ingegneria Industriale Universita' degli Studi di Parma Parco Area delle Scienze 181/A I-43100 Parma, Italia Fax: +39-0521-905705

E-mail: frugoli@ied.eng.unipr.it Http://www.unipr.it/

\section{Andrea Galimberti}

Dipartimento di Ingegneria Industriale

Universita' degli Studi di Parma

Parco Area delle Scienze 181/A

I-43100 Parma, Italia

Fax: +39-0521-905705

E-mail: galimba@ied.eng.unipr.it

Http://www.unipr.it/

\section{Dominik Henrich}

Embedded Systems and Robotics (RESY)

Faculty of Informatics

University of Kaiserslautern

Building 48

D-67653 Kaiserslautern, Germany

Fax: +49-631-205-2649

E-mail: henrich@informatik.uni-kl.de

Http: //resy.informatik.uni-kl.de/

\section{Shinichi Hirai}

Department of Robotics

Ritsumeikan University

Kusatsu

Shiga 525-8577, Japan

Fax: +81-77-561-2665

E-mail: hirai@se.ritsumei.ac.jp

Http://www.ritsumei.ac.jp/se/ hirai/

index-e.html

\section{Karl-Friedrich Kämper}

BJB GmbH \& Co.KG

Werler Strasse 1

D-59755 Arnsberg, Germany

Fax: +49-2932-982-390

E-mail: marika.pitts@bjb.de

Http://www.bjb.de/ 


\section{Kazuhiro Kosuge}

Department of Machine Intelligence and Systems Engineering

Tohuku University

Aoba-yama 01, Aramaki, Aobaku

Sendai 980-8579, Japan

Fax: +81-22-217-6915

E-mail: kosuge@irs.mech.tohoku.ac.jp

Http://www.irs.mech.tohoku.ac.jp/

\section{Kostas J. Kyriakopoulos}

Control Systems Laboratory

Mechanical Engineering Department

National Technical University of

Athens

9 Herroon Polytechniou Str.,

Zografou Athens 15700, Greece

Fax: +30-1-772-3657

E-mail: kkyria@central.ntua.gr

\section{Yoshinaga Maruyama}

Techniques Development of the

Distribution Department

Kyushu Electric Power Co.

2-1-82, Watanabe-dori Chuo-ku

Fukuoka 810-8720, Japan

Fax: +81 927125236

E-mail:

Yoshinaga_Maruyama@kyuden.co.jp Http://www.kyuden.co.jp/Distribution/E nglish/Index2-Distribution.html

\section{Brenan J. McCarragher}

Department of Engineering

Faculty of Engineering and Information Technology

Australian National University

Canberra, ACT 0200, Australia

Fax: + 61-2-6249-0506

E-mail:

Brenan.McCarragher@anu.edu.au

Http://spigot.anu.edu.au/people/brenan /home.html

\section{Eiichi Ono}

Intelligent Systems Division

Electrotechnical Laboratory AIST, MITI

1-1-4, Umezono

Tsukuba, Ibaraki, 305-8568, Japan

Fax: +81-298-54-5971

E-mail: eono@etl.go.jp

Http://www.etl.go.jp/ eono/

\section{Axel Remde}

Institute for Process Control and

Robotics (IPR)

Computer Science Department

Universität Karlsruhe (TH)

Kaiserstrasse 12

D-76128 Karlsruhe, Germany

Fax: +49-721-608-7141

E-mail: Remde@ira.uka.de

Http://wwwipr.ira.uka.de/ remde/

\section{Caterina Rizzi}

Dipartimento di Ingegneria Industriale

Universita' degli Studi di Parma

Parco Area delle Scienze 181/A

I-43100 Parma, Italia

Fax: +39-0521-905705

E-mail: rizzi@ied.eng.unipr.it

Http://www.unipr.it/

\section{Herbert Tanner}

Control Systems Laboratory

Mechanical Engineering Department

National Technical University of

Athens

9 Herroon Polytechniou Str.,

Zografou Athens 15700, Greece

Fax: +30-1-772-3657

E-mail: htanner@central.ntua.gr 


\section{Takahiro Wada}

Arimoto and Kawamura Laboratory Department of Robotics

Faculty of Science and Engineering Ritsumeikan University

1-1-1 Noji-higashi, Kusatsu

Shiga, 525-8577, Japan

Fax: +81-77-561-2811

E-mail: wachan@ robot.club.ne.jp Http://www.geocities.co.jp/BerkeleyLabo/1913/index-e.html

\section{Heinz Wörn}

Institute for Process Control and Robotics (IPR)

Computer Science Department

Universität Karlsruhe (TH)

\section{Kaiserstrasse 12}

D-76128 Karlsruhe, Germany

Fax: +49-721-608-7141

E-mail: Woern@ira.uka.de

Http://wwwipr.ira.uka.de/

\section{Hidehiro Yoshida}

Production Engineering Laboratory

Corporate Production Engineering

Division

Matsushita Electric Industrial Co., Ltd

2-7, Matsuba-cho

Kadoma, Osaka, 571-8502, Japan

Fax: +81-6-6905-4518

E-mail: hyoshida@labo.ped.mei.co.jp

Http://www.labo/pel 
Chapter 1

\section{Introduction}




\section{Section 1.1}

\section{Motivation}

\section{H. Wörn}

Besides the usage of industrial robots in classical fields of application, e.g., welding, painting and handling, applying robotic systems in additional areas like assembly, machining and measuring is gaining more and more importance. Many research and development projects aiming at the exploitation of these new application areas have been carried out and their results reflect in innovative products and visionary future perspectives.

Under this framework of innovative robot applications, many interesting and challenging topics are related to the handling and processing of deformable objects ${ }^{1}$. By observing the manual processing of such objects, we conclude that a successful task execution especially relies on the following components:

- $\quad$ visual and tactile information,

- multi-fingered hand,

- two-armed corporation,

- $\quad$ prior knowledge and experience.

Developing an ideal and versatile robot system for the handling of flexible objects therefore requires to address the following topics:

- $\quad$ sensor data processing and multi-sensor fusion,

- development of robust and dexterous grippers,

- multi-robot corporation,

- $\quad$ modeling and simulation,

1 In this context, an object is called "deformable" if its deformation must be considered for the manipulation process. 
- $\quad$ artificial intelligence.

All of these topics are current research topics, causing the robot handling of flexible materials to be a very challenging field from an academic and scientific point of view.

Since flexible materials are found in almost every industrial product, automated handling of such materials becomes significant from an industrial and economic point of view, too. Two examples can demonstrate this importance: The complete textile industry deals with the processing of highly deformable objects. The price for cloths is mainly depends on the costs of handling and processing the textiles. Though many common tasks like the lapping, (un)folding and positioning have been addressed in many robotic research works and a large variety of prototype systems has been set up, the large-scaled application of robotic handling systems has not yet been achieved. However, the problem of automated textile handling is still on the agenda and the work is being continued, as reflected in different contributions of this book.

The second example is the world of the automotive industry, which is wellknown to be one of the most important branches in most industrial countries. Among the large variety of product components to be processed and assembled, many are partly or highly flexible. Though the level of automation and robot implementation is very high in the production, the number of robots is, though increasing, rather small in the final assembly. This causes the assembly costs to be one of the most dominant factors in the final product price.

Because of the large variety of tasks and materials and the automation-friendly environment, the automotive industry is an almost ideal branch to develop, inves tigate and implement new robot applications. Accordingly, many assembly tasks involving flexible materials, such as, the assembly of hoses, O-ring seals and rubber sealing profiles have been addressed, resulting in robotic prototype systems. For the future, a significant increase of robot applications in the handling of flexible materials can be expected, driven by the high general effort for automating assembly operations in this branch of industry.

When thinking about automated handling, there are two principal ways in dealing with the non-constant object shape. One way is to develop a highly sophisticated, special-purposed robot end-effectors, reflecting the mechanical workpiece properties. Here, most of the effort is required for the hardware design, while using established traditional methods for programming the robot. This approach is proven to meet both key requirements of short cycle time and high reliability.

The other way is to use a more human-like approach, i.e., to keep the design of the hardware as general and flexible (but not necessarily simple and cheap) as possible and to spend more effort for aspects as sensors, sensor data processing, simulation and prediction. The major advantage of this approach is found in the high flexibility and in the possibility of adopting the system to varying tasks, as demonstrated in the human behavior.

Although the flexibility and generality displayed in this second approach are very attractive, it requires to cope with several difficult issues given above, from sensor data fusion to artificial intelligence. The problems related to these topics 
could not yet been satisfying solved for many tasks less complicated than the handling of flexible materials. In opposite to this, the development of special-purposed hardware has been industrial everyday work for decades. Accordingly, experience and knowledge are widely available here, causing the application of dedicated hardware solutions to be more feasible from a practical point of view.

As the degree of automation in the assembly increases, the number of robot applications in the handling of flexible materials can also be expected to grow. While the first step is the large-scaled integration of special-purpose end-effectors and other hardware tools, the increasing number of complex sensor applications (caused by the demand for flexible and fault-tolerant robotic systems) and the general increase of scientific knowledge will stimulate the introduction of sophisticated and flexible handling systems. This kind of development, starting with hardware solutions dedicated to the economic solution of well-defined problems and leading to more flexible and general systems, can be found in many variations in the industrial his tory. Only one of many examples is the machine-tools development, starting with simple milling machines and lathes over highly specialized onepurpose equipment to the flexible NC-technology.

One should note that finding a technically and economically significant solution to industrial problems is not a simple one-dimensional problem, but depends on many factors, including technological, social and political aspects, and change in any of them may affect the answer to the initial question as well. Therefore it is wise not to focus on one approach only that seems to be straight-forward, but to investigate a larger variety of principles and methods, even if the industrial application seems to be somewhat futuristic in some cases.

For the automated handling of flexible materials, the aim of this book is to give a survey of both the state of the art and technology. Some of the contributions in this book describe innovative applications of robot systems which could already be established or are expected to have a break-through within the next few years. Others deal with the handling of flexible materials from a more fundamental point of view, highlighting milestones which could be achieved and problems which are under research and will have to be solved in the framework of a long-term perspective. 


\section{Section 1.2}

\section{About this Book}

\section{Henrich}

Besides the work in the field of manipulating rigid objects, currently, there are several research and development activities going on in the field of manipulating non-rigid or deformable objects. Several papers have been published on international conferences in this field from various projects and countries. But there has been no comprehensive work which provides both a representative overview of the state of the art and identifies the important aspects in this field.

Thus, we collected these activities and invited the corresponding working groups to present an overview of their research. Altogether, nineteen authors coming from Japan, Germany, Italy, Greece, United Kingdom, and Australia contributed to this book. Their research work covers all the different aspects that occur when manipulating deformable objects. The contributions can be characterized and grouped by the following four aspects:

- object modeling and simulation,

- planning and control strategies,

- collaborative systems, and

- $\quad$ applications and industrial experiences.

In the following, we give a short motivation and overview of the single chapters of the book.

The simulation of deformable objects is one way to approach the problem of manipulating these objects by robots. Based on a physical model of the object and the occurring constraints, the resulting object shape is calculated. In Chapter 2, Hirai presents an energy-based approach, where the internal energy under the geometric constraints is minimized. Frugoli et al. introduce a force-based approach, where the forces between discrete particles are minimized meeting given constraints. Finally, Remde and Henrich extend the energy-based approach to plastic 
deformation and give a solution of the inverse simulation problem.

Even if the object behavior is predicted by simulation, there is still the question of how to control the robot during a single manipulation operation. An additional question is how to retrieve an overall plan for the concatenated manipulation operations. In Chapter 3, Wada investigates the control problems when positioning multiple points of a planar deformable object. McCarrager proposes a control scheme exploiting the flexibility, rather than minimizing it. Abegg et al. use a simple contact state model to describe typical assembly tasks and to derive robust manipulation primitives. Finally, Ono presents an atomatic sewing system and suggests a strategy for unfolding fabric.

In several manipulation tasks, it is reasonable to apply more than one robot. Especially in cases, where the deformable object has to take a specific shape. Since the robots working at the same object are influencing each other, different control algorithms have to be introduced. In Chapter 4, Yoshida and Kosuge investigates this problem for the task of bending a sheet of metal and exploits the relation ship between the static object deformation and the bending moments. Tanner and Kyriakopoulos regard the deformable object as underactuated mechanical system and make use of the existence of non-holonomic constraints. Both approaches model the deformable object as finite elements.

All of the above aspects have their counterpart in different applications and industrial experiences. In Chapter 5, Rizzi et al. present test cases and applications of their approach to simulate the manipulation of fabric, wires, cables, and soft bags. Buckingham and Graham give an overview of two European projects processing white fish including locating, gripping, and deheading the fish. Maruyama outlines the three development phases of a robot system for performing outage-free maintenance of live-line power supply in Japan. Finally, Kämper presents the development of a flexible automatic cabling unit for the wiring of long-tube lighting with plug components. 
Chapter 2

Material Modeling and Simulation 
Section 2.1

Energy-Based Modeling of Deformable Linear Objects

S. Hirai 
Section 2.2

Discrete Element Approach for Non-Rigid Material Modeling

G. Frugoli, A. Galimberti, C. Rizzi, and M. Bordegoni 
Section 2.3

Direct and Inverse Simulation of Deformable Linear Objects

A. Remde and D. Henrich 
Chapter 3

\section{Planning and Control Strategies}


Section 3.1

Indirect Simultaneous Positioning of Extensible Deformable Objects

T. Wada 
Section 3.2

A Hybrid Position / Force Approach to the Exploitation of Elasticity in Manipulation

B. J. Mc Carragher 
Section 3.3

Force- and Vision-Based Detection of Contact State Transitions

F. Abegg, A. Remde, and D. Henrich 
Section 3.4

Automated Sewing System and Unfolding Fabric

E. Ono 
Chapter 4

Collaborative Systems 
Section 4.1

Manipulation of Sheet Metal by Dual Manipulators

H. Yoshida and K. Kosuge 
Section 4.2

A Manipulated Deformable Object as an Underactuated Mechanical System

H. G. Tanner and K. J. Kyriakopoulos 
Chapter 5

Applications and Industrial Experiences 
Section 5.1

Simulation of Non-Rigid Materials Handling

C. Rizzi, M. Bordegoni, and G. Frugoli 
Section 5.2

\section{Robotics for Deheading White Fish}

R. Buckingham and A. Graham 
Section 5.3

Application of LLW Robots to Distribution Lines

Y. Maruyama 
Section 5.4

Flexible Automatic Wiring of Long-Tube Lighting and Service Cabinet Modules

K.-F. Kämper 